Document downloaded from:

http://hdl.handle.net/10251/74041

This paper must be cited as:

Tur Valiente, M.; García, E.; Baeza González, LM.; Fuenmayor Fernández, FJ. (2014). A 3D absolute nodal coordinate finite element model to compute the initial configuration of a railway catenary. Engineering Structures. 71:234-243. doi:10.1016/j.engstruct.2014.04.015.

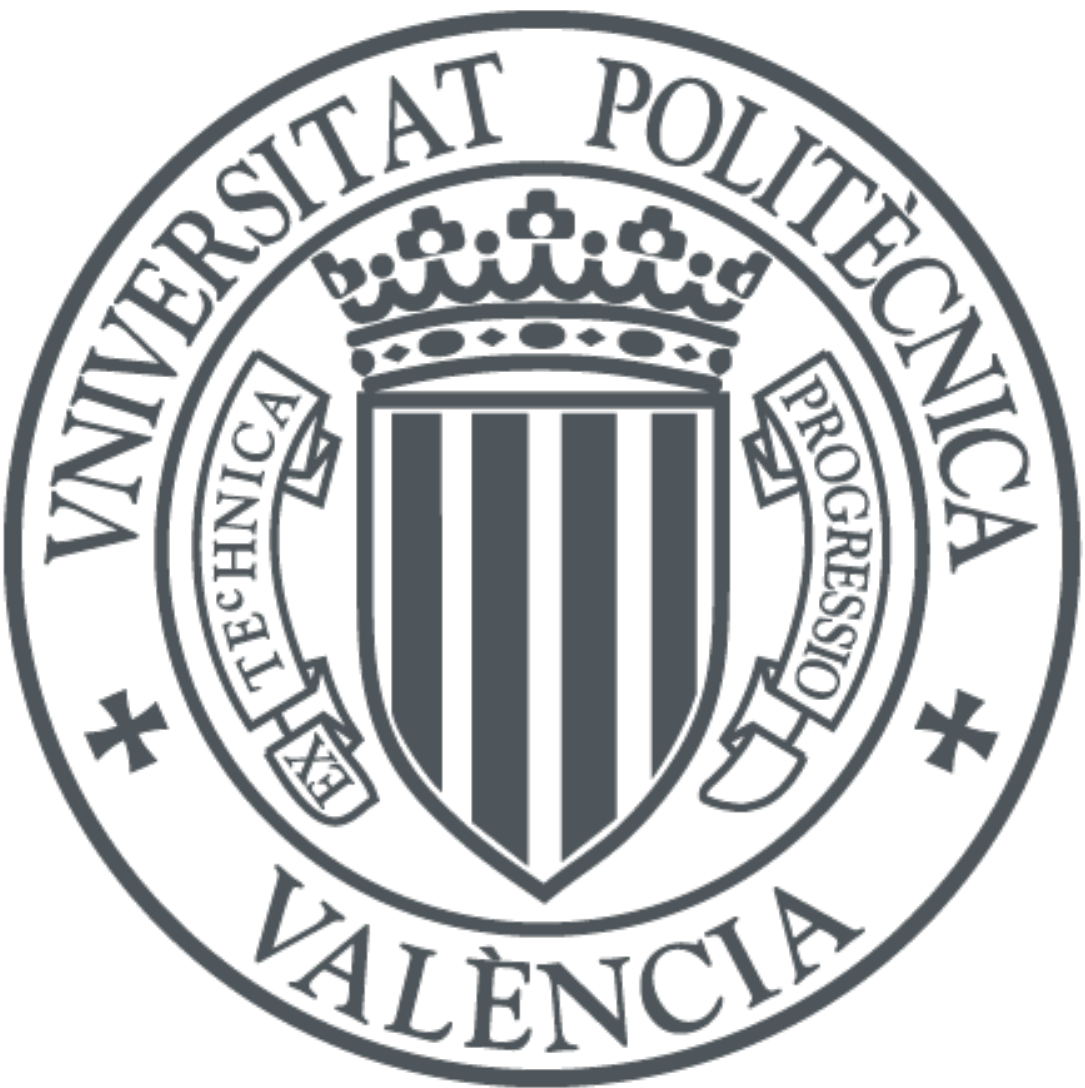

The final publication is available at

http://dx.doi.org/10.1016/j.engstruct.2014.04.015

Copyright Elsevier

Additional Information 


\title{
A 3D absolute nodal coordinate finite element model to compute the initial configuration of a railway catenary
}

\author{
M. Tur ${ }^{\mathrm{a}, *}$, E. García ${ }^{\mathrm{b}}$, L. Baeza ${ }^{\mathrm{a}}$, F.J. Fuenmayor ${ }^{\mathrm{a}}$ \\ ${ }^{a}$ Centro de Investigación de Tecnología de Vehículos, Departamento de Ingeniería \\ Mecánica y de Materiales, Universitat Politècnica de València, Camino de Vera s/n, \\ 46022 Valencia, Spain \\ ${ }^{b}$ Patentes Talgo S.L., Paseo del Tren Talgo 3, 28290 Las Matas, Spain
}

\begin{abstract}
In this paper we propose a method of finding the initial equilibrium configuration of cable structures discretized by finite elements applied to the shape-finding of the railway overhead system. Absolute nodal coordinate formulation finite elements, which take into account axial and bending deformation, are used for the contact and messenger wires. The other parts of the overhead system are discretized with non-linear bars or equivalent springs. The proposed method considers the constraints introduced during the assembly of the catenary, such as the position of droppers, cable tension, height of the contact wire, etc. The main result of the shape-finding problem is the computation of the length of droppers. A comparison of the results obtained for reference catenaries in the bibliography is also included.
\end{abstract}

Keywords: catenary, shape-finding, absolute nodal coordinate, overhead system

\section{Introduction}

The overhead system is responsible for transmitting electrical power to the locomotive in an uninterrupted flow of current and consists of two main components: the catenary and the pantograph. The former is the static structure built over the railroad tracks to carry the contact wire. The pantograph is the mechanism attached to the train that presses against the contact wire

\footnotetext{
*Corresponding author. Tel:+34 963877621 Fax: +34 963877629

Email address: matuva@mcm.upv.es ( M. Tur)
} 
in such a way as to achieve a permanent contact that ensures power transmission with the lowest possible contact force in order to minimize wear on the system.

Simulating the dynamic interaction between the pantograph and the catenary is a useful tool in the design of more efficient catenary systems. With these tools designers can analyze, for example, the effects of the catenary geometry or the properties of the cables on the contact force between pantograph and catenary. The number of codes developed in recent years [1-16] is an example of just how important simulation is becoming in this field. A common feature of most of the codes is that the cables are modeled using the finite element method, mainly using prestressed beam formulations, like the Euler-Bernoulli or Timoshenko beam theories. The absolute nodal coordinate formulation (ANCF) proposed by Shabana $[17,18]$ is a cable model also used in dynamic simulations of the catenary system, for example in [15, 16]. The pantograph is usually simplified as a lumped mass model, although some codes incorporate a more complex multibody model [19, 20].

One concern of finite element models of cable structures like the catenary system is how to obtain the initial configuration, i.e. the undeformed length of the elements and the global position of every node in the mesh that is in static equilibrium with external loads. This is usually referred to in the bibliography as the shape-finding or shape forming of the structure. This is a complex problem since cables are extremely flexible structures with highly non-linear behavior and undergo large displacements before attaining the equilibrium configuration from an unknown reference configuration. The designer must therefore obtain the initial equilibrium configuration, taking into account how the system is assembled in practice and the geometric constraints imposed. Although little attention has been paid in the literature to the shape-finding of the catenary system, it is a well-known and challenging problem in the design of other cable structures, such as light large-area roofs or cable-supported bridges [21-26]. As pointed out in [27, 28], the initial configuration of the catenary can strongly influence the results of the dynamic simulation.

The aim of this work is to propose a method of computing the initial configuration of cable structures modeled by the finite element method using ANCF cable elements. A review of shape-finding methods can be found in [21]. The non-linear displacement method [22, 23] and the force density method [24-26] are the two most commonly used formulations. The method proposed in this work can be considered a variation of the non-linear dis- 
placement method, and even though it is a general method, we applied it to finding the initial configuration of a railway catenary. The method takes into account any constraints that may appear during the assembly of the catenary. It can also be used to compute the length of the droppers in order to obtain a given height for the contact wire.

Some algorithms have been proposed in recent years to compute the equilibrium position of the catenary and the length of the droppers [28-30]. However, the present paper uses a different method to solve the shape-finding problem as well as a different model for the wires. A Euler-Bernouilli beam is used in [29] and special catenary elements are used in [28, 30]. [31] reviews the different special elements used in the literature to model wires. Although classical finite element techniques, such as the two-node truss element or isoparametric elements, may be used in the literature, other improved formulations are preferred. These are based on a mathematical description of the deformed wire, as in the parabolic approximation element [REFS], or the more popular catenary element $[28,31-34]$. In this paper we use the absolute nodal coordinate formulation (ANCF) $[16-18,35]$ element to model the messenger and contact wires and traction bar elements for the droppers. The ANCF element accounts for the bending and axial deformations of the wires. The formulation is 3D and can consider large deformations. The ANCF element has $C^{1}$ continuity, which can be important in the dynamic simulation of the contact between the contact wire and the pantograph. The ANCF element was used in [15] to model a $2 \mathrm{D}$ catenary and in $[16,36]$ to construct a $3 \mathrm{D}$ railway catenary.

Concerning the railway catenary, in the last years some algorithms have been proposed to compute the equilibrium position and obtain the length of the droppers [28-30]. The differences between these works and the present paper are the method used to solve the shape-finding problem as well as the model used for the wires. A Euler-Bernouilli beam is used in [29] or special catenary elements are used in $[28,30]$. In [31] there is a review of different special elements used in the literature to model wires. Although classical finite element techniques like the two-node truss element or isoparametric elements could be used in the literature other improved formulation are preferred. These formulations are based on a mathematical description of the deformed wire, like the parabolic approximation element [37] or the more popular catenary element $[28,31-34]$. In this paper we use the absolute nodal coordinate formulation (ANCF) $[16-18,35]$ element to model the messenger and contact wires and traction bar elements for the droppers. The 
ANCF element accounts for the bending and axial deformations of the wires. The formulation is 3D and large deformation is considered. ANCF element has $C^{1}$ continuity which can be important in the dynamic simulation of the contact between the contact wire and the pantograph. The ANCF element was used in [15] to model a $2 \mathrm{D}$ catenary and in $[16,36]$ to construct a $3 \mathrm{D}$ railway catenary.

The paper is organized as follows. In Section (2) the main components of the railway catenary are outlined. The formulation of the ANCF element is reviewed in Section (3). In Section (4) we detail the proposed formulation to find the initial configuration of the catenary. Two methods are proposed to obtain the desired position of the contact wire and compute the length of the droppers and the initial configuration. In Section (5) the proposed formulation is used to solve some reference problems and compare the results with those in the bibliography. The conclusions are summarized in Section (6).

\section{Description of a railway catenary}

A railway catenary is depicted in Figure (1). The cables include the messenger or carrier wire, the contact wire, the droppers and may include stitch wires. Masts, brackets and registration arms are the structural elements that support the whole system. The messenger wire is supported at regular intervals on brackets at a certain height over the track. The main function of the messenger wire is to hold the contact wire (to which it is attached at predefined intervals by droppers), which transmits the electrical current to the pantograph. The droppers are fixed to the contact ande messenger wires by clamps, providing the whole catenary system with a certain stiffness. The stitch wire modifies the stiffness of the catenary near the supports. As in the case of the droppers, clamps are used to attach the stitch wires to the messenger and contact wires.

The initial configuration of the cables largely depends on how the assembly of the catenary system is performed. Here we briefly outline the main features of the assembly that are important for the definition of the shapefinding method proposed in this work. The finite element model must fullfil the constraints introduced during the stringing of the catenary.

First, the external structural elements (masts and brackets) are installed along the track. The main messenger and contact wires are then tensioned in a number of spans. The messenger wire is connected to the brackets and 


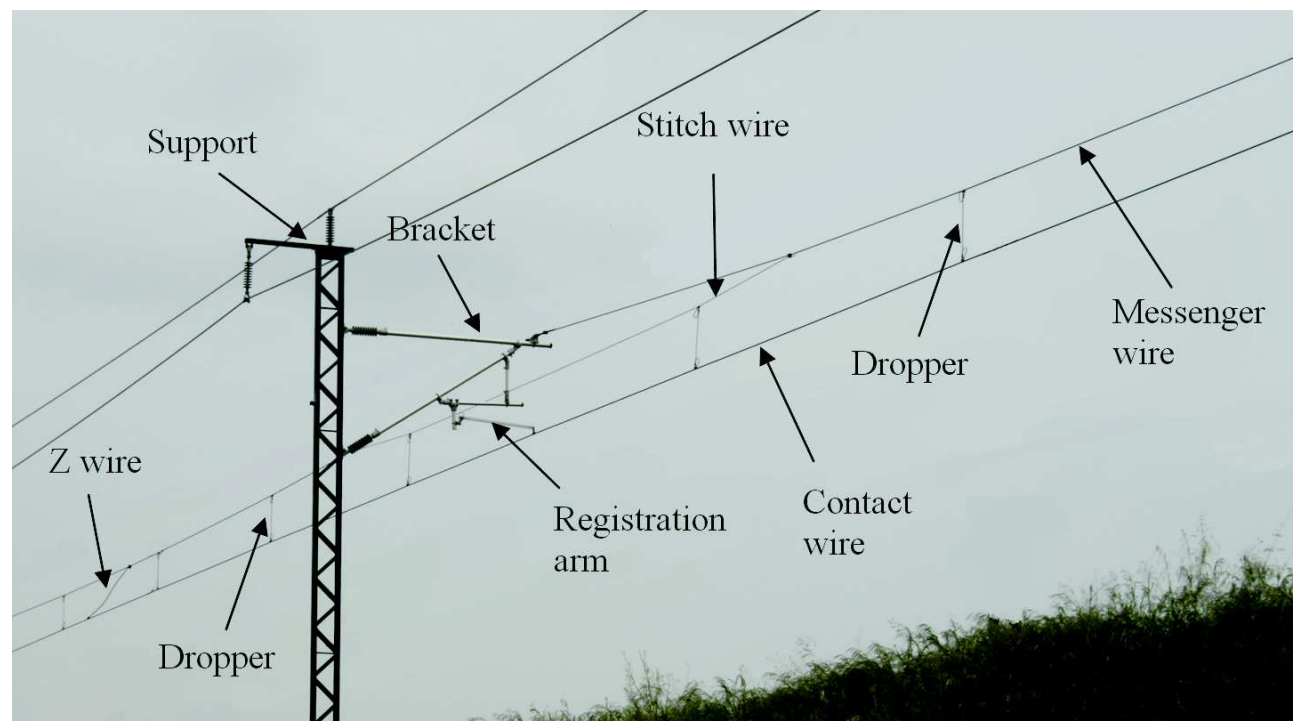

Figure 1: Picture of a high-speed train catenary.

the contact wire is connected to the registration arm. The tension is held constant with the help of systems mounted on the first and last masts. A stagger is defined along the track for the contact wire and optionally for the messenger wire. The stitch wire is then mounted and a predefined tension is applied. Finally, the droppers are installed at predefined points along the track in each span. Each dropper has a given length in order to obtain the correct height of the contact wire at the connection points. The catenary can be defined with or without presag. In the former case, the height of the contact wire is defined as a predefined function with zero sag at the first and last dropper in the span and maximum sag at the central dropper. In the latter, the height of the wire at the dropper connection points is constant.

The finite element model of the catenary used in this work is shown in Figure (2). The nodes of the finite element mesh are shown as circles. The model contains different types of two-node elements. The ANCF beam element is used to model both the messenger and contact wires and allows for axial and bending deformation in the wires. The droppers are modeled as non-linear bar elements that can only transmit traction forces. The registration arm is a single straight bar element. The messenger wire and registration arm supports can be defined by a simple constraint of the displacement or can be replaced by equivalent springs and dampers. In the following Sec- 


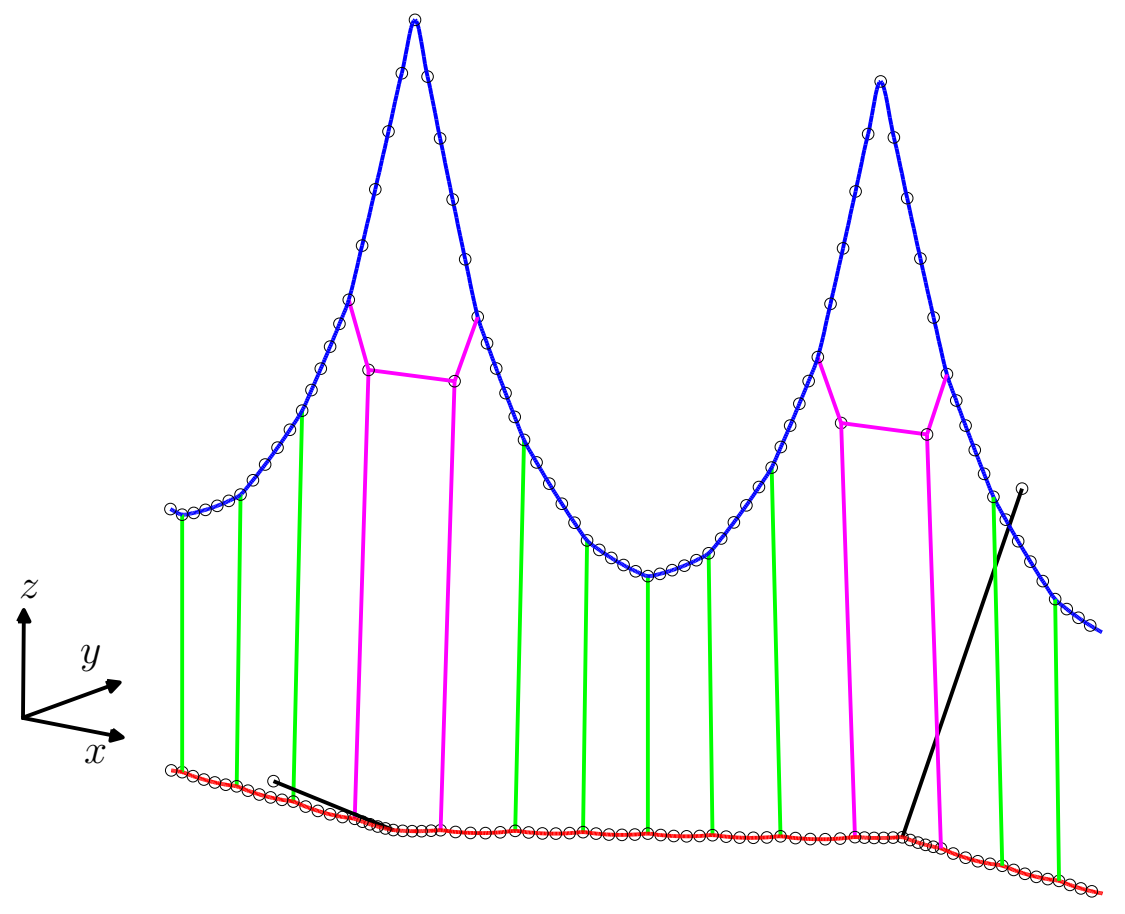

Figure 2: Finite element model of the catenary.

tion the main aspects of the finite element formulation used in this work are described.

\section{Finite element formulation}

As pointed out above, the wires undergo large deformations when the catenary is assembled, which means that a nonlinear finite element formulation has to be used. In this work two types of elements are used. The first is a beam element based on the absolute nodal coordinate formulation. This element was introduced in $[17,18]$. In 3D problems it has 6 degrees of freedom per node (coordinates and gradients) and it accounts for axial and bending deformations when computing the strain energy. The main difference between this element and the classical nonlinear beam formulation is the use of gradients instead of rotational degrees of freedom. The element's performance was analyzed in $[16,35]$ and compared with the classical formulation in [38]. The second element is a non-linear two-node bar. 


\subsection{Absolute nodal coordinate element}
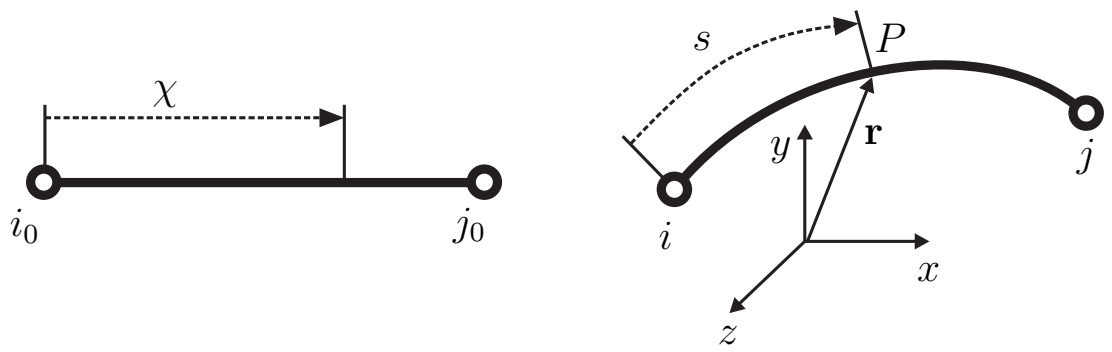

Figure 3: Undeformed and deformed configurations of the ANCF element.

Here we used the formulation presented in [16, 17]. Figure (3) shows the undeformed and deformed configurations of an element with two nodes $i$ and $j$. We define the nodal degree of freedom vector of the element that contains the displacements and the gradients as:

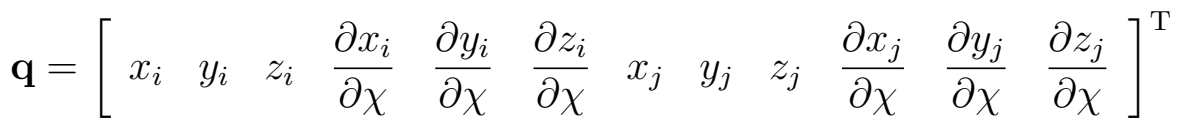

where $\chi$ is the local coordinate in the undeformed configuration ranging from 0 to the element initial length $l_{0}$. The position vector in the deformed configuration $\mathbf{r}$ is a cubic polynomial and can be interpolated using the shape functions matrix as:

$$
\mathbf{r}=\left\{\begin{array}{c}
a_{0}+a_{1} \chi+a_{2} \chi^{2}+a_{3} \chi^{3} \\
b_{0}+b_{1} \chi+b_{2} \chi^{2}+b_{3} \chi^{3} \\
c_{0}+c_{1} \chi+c_{2} \chi^{2}+c_{3} \chi^{3}
\end{array}\right\}=\mathbf{S} \mathbf{q}
$$

Taking into account the definition of the normalized local coordinate $\xi=\chi / l_{0}$ and the $C^{1}$ continuity between elements, the interpolation matrix is defined as:

$$
\begin{aligned}
\mathbf{S}= & {\left[\begin{array}{cccccccccccc}
S_{1} & 0 & 0 & S_{2} & 0 & 0 & S_{3} & 0 & 0 & S_{4} & 0 & 0 \\
0 & S_{1} & 0 & 0 & S_{2} & 0 & 0 & S_{3} & 0 & 0 & S_{4} & 0 \\
0 & 0 & S_{1} & 0 & 0 & S_{2} & 0 & 0 & S_{3} & 0 & 0 & S_{4}
\end{array}\right] } \\
& S_{1}(\xi)=1-3 \xi^{2}+2 \xi^{3} \\
& S_{2}(\xi)=l_{0}\left(\xi-2 \xi^{2}+\xi^{3}\right) \\
& S_{3}(\xi)=3 \xi^{2}-2 \xi^{3} \\
& S_{4}(\xi)=l_{0}\left(\xi^{3}-\xi^{2}\right)
\end{aligned}
$$


The strain energy of the element is computed from the contribution of axial and bending deformations respectively as:

$$
U^{\mathrm{C}}=\frac{1}{2} \int_{0}^{l_{0}}\left(E A \varepsilon_{\mathrm{L}}^{2}+E I \kappa^{2}\right) d \chi
$$

where $E$ is the Young's modulus, $A$ is the area and $I$ is the moment of inertia, $\varepsilon_{\mathrm{L}}$ is the axial deformation and $\kappa$ the curvature. The axial deformation is computed as:

$$
\varepsilon_{\mathrm{L}}=\frac{1}{2}\left(\mathbf{r}^{\prime \mathrm{T}} \mathbf{r}^{\prime}-1\right)=\frac{1}{2}\left(\mathbf{q}^{\mathrm{T}} \mathbf{S}^{\prime \mathrm{T}} \mathbf{S}^{\prime} \mathbf{q}-1\right)
$$

where the abbreviation for the spatial derivative is used:

$$
\mathbf{r}^{\prime}=\frac{\partial \mathbf{r}}{\partial \chi}=\frac{1}{l_{0}} \frac{\partial \mathbf{r}}{\partial \xi} \quad \mathbf{S}^{\prime}=\frac{\partial \mathbf{S}}{\partial \chi}=\frac{1}{l_{0}} \frac{\partial \mathbf{S}}{\partial \xi}
$$

The curvature can be approximated using the following equation:

$$
\kappa=\left|\mathbf{r}^{\prime \prime}\right|=\sqrt{\mathbf{q}^{\mathrm{T}} \mathbf{S}^{\prime \prime} \mathbf{S}^{\prime \prime} \mathbf{q}}
$$

Note that this equation is valid for low axial strains such as that found in the catenary wires.

The equivalent nodal force vector due to axial and bending deformation can be computed from the derivative of the strain energy (equation (4)) with respect to the nodal coordinates:

$$
\mathbf{f}_{e}^{\mathrm{C}}\left(\mathbf{q}, l_{0}\right)=\frac{\partial U^{\mathrm{C}}}{\partial \mathbf{q}}=\frac{1}{2} \int_{0}^{l_{0}}\left(E A \varepsilon_{\mathrm{L}} \frac{\partial \varepsilon_{\mathrm{L}}}{\partial \mathbf{q}}+E I \frac{\partial \kappa^{2}}{\partial \mathbf{q}}\right) d \chi
$$

Details of the formulation and the computation of the integrals can be found in [18]. Assuming that gravity is acting in $z$ direction, the nodal equivalent force due to gravity can be written as:

$$
\begin{aligned}
\mathbf{f}_{g}^{\mathrm{C}}\left(l_{0}\right) & =\int_{0}^{l_{0}} \mathbf{S}^{\mathrm{T}}\left\{\begin{array}{llll}
0 & 0 & -g \rho A
\end{array}\right\}^{\mathrm{T}} d \chi \\
& =-g \rho A l_{0}\left\{\begin{array}{llllllllllll}
0 & 0 & \frac{1}{2} & 0 & 0 & \frac{l_{0}}{12} & 0 & 0 & \frac{1}{12} & 0 & 0 & -\frac{l_{0}}{12}
\end{array}\right\}^{\mathrm{T}}
\end{aligned}
$$

where $\rho$ is the density.

The equivalent nodal force vector is obtained by adding equations (8) and (9). Note that the force is a function of the deformed nodal coordinates and the initial length of the element:

$$
\mathbf{f}^{\mathrm{C}}\left(\mathbf{q}, l_{0}\right)=\mathbf{f}_{e}^{\mathrm{C}}\left(\mathbf{q}, l_{0}\right)+\mathbf{f}_{g}^{\mathrm{C}}\left(l_{0}\right)
$$




\subsection{Discontinuity in axial force}

As pointed out above, the ANCF element has $C^{1}$ continuity. This means that the gradients of the position are continuous between elements and therefore it is the axial strain. This is a drawback of this type of element if one wire is connected to another (for example, the stitch wire with the messenger wire in Figure (2) or the connection between elements $e 1$ and $e 2$ in Figure (4)). In this particular case the axial tension will be discontinuous and the element is not able to account for this.

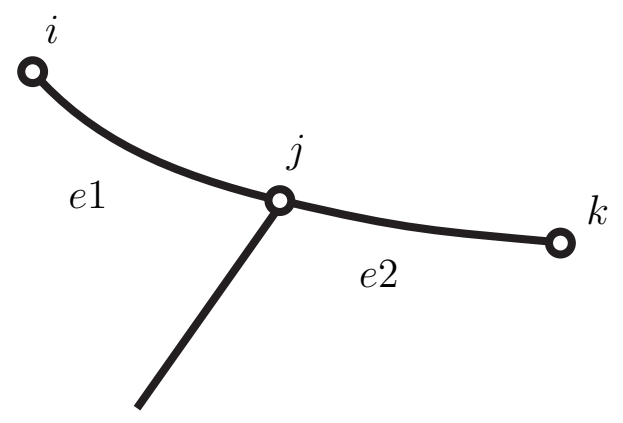

Figure 4: Connection of elements with discontinuous axial deformation.

In order to avoid problems with discontinuities in the axial force transmission, an improved element can be defined. A new degree of freedom $\alpha$ is added to element $e 2$ in Figure (4). Without loss of generality we assume that the new degree of freedom $\alpha$ is associated with the first node $j$ of element $e 2$. The coordinates of the elements $e 1$ and $e 2$ are defined as:

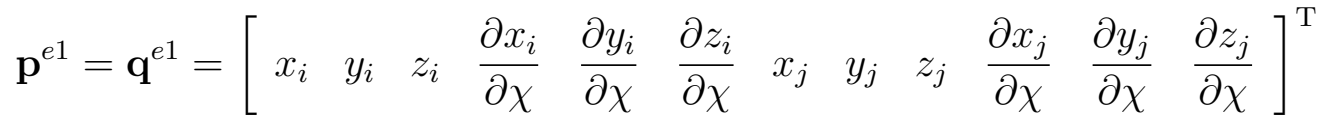

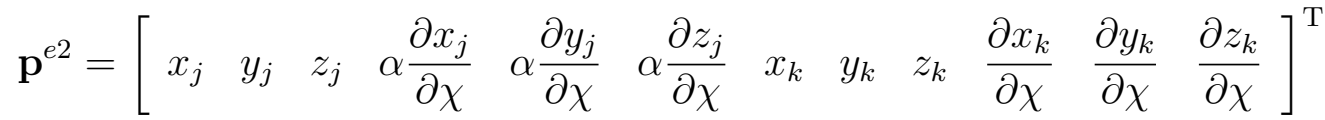

Note that the gradients of node $j$ of element $e 2$ are modified by a factor $\alpha$. This factor allows the element to have a jump in the axial strain in the node $j$ while keeping continuity in the slope. The continuity is obtained because the direction of the cable element depends only on the ratios of the components of Vector $\mathbf{p}, \mathrm{p}_{4}^{e 2} / \mathrm{p}_{3}^{e 2}=\mathrm{p}_{4}^{e 1} / \mathrm{p}_{3}^{e 1}, \mathrm{p}_{5}^{e 2} / \mathrm{p}_{3}^{e 2}=\mathrm{p}_{5}^{e 1} / \mathrm{p}_{3}^{e 1}$ and $\mathrm{p}_{5}^{e 2} / \mathrm{p}_{4}^{e 2}=\mathrm{p}_{5}^{e 1} / \mathrm{p}_{4}^{e 1}$, which are independent of the new coordinate $\alpha$. Therefore, the same direction is obtained for both elements at the connection point. 
Similarly to the ANCF element, the equivalent nodal force vector due to axial and bending deformation can be computed from the derivative of the strain energy (Equation (4)) with respect to the nodal coordinates. In this case we obtain:

$$
\mathbf{f}_{e}^{\mathrm{ST}}\left(\mathbf{q}, \alpha, l_{0}\right)=\left\{\begin{array}{c}
\frac{\partial U^{\mathrm{C}}}{\partial \mathbf{q}} \\
\frac{\partial U^{\mathrm{C}}}{\partial \alpha}
\end{array}\right\}=\left\{\begin{array}{c}
\frac{\partial U^{\mathrm{C}}}{\partial \mathbf{p}} \frac{\partial \mathbf{p}}{\partial \mathbf{q}} \\
\frac{\partial U^{\mathrm{C}}}{\partial \mathbf{p}} \frac{\partial \mathbf{p}}{\partial \alpha}
\end{array}\right\}
$$

\subsection{Nonlinear bar element}

The nonlinear bar element is a straigth line with two nodes $i$ and $j$ and six degrees of freedom (the global coordinates of each node). The strain energy can be computed from Equation (4), taking into account only the axial deformation term (i.e. $I=0$ ). The axial deformation can be computed as:

$$
\varepsilon_{\mathrm{L}}=\frac{l_{d}^{2}-l_{0}^{2}}{2 l_{0}^{2}}
$$

where $l_{0}$ is the undeformed length and $l_{d}$ is the deformed length of the element

$$
l_{d}^{2}=\left(x_{j}-x_{i}\right)^{2}+\left(y_{j}-y_{i}\right)^{2}+\left(z_{j}-z_{i}\right)^{2}
$$

The equivalent nodal force vector due to strain $\mathbf{f}_{e}^{\mathrm{B}}\left(\mathbf{q}, l_{0}\right)$ is computed using Equation (8) and the force due to gravity from Equation (9) to obtain:

$$
\mathbf{f}_{g}^{\mathrm{B}}\left(l_{0}\right)=-\frac{g \rho A l_{0}}{2}\left\{\begin{array}{llllll}
0 & 0 & 1 & 0 & 0 & 1
\end{array}\right\}^{\mathrm{T}}
$$

Again, the force introduced by the bar element is the contribution of elastic deformation and gravitational forces and is a function of the deformed coordinates $\mathbf{q}$ and the initial length:

$$
\mathbf{f}^{\mathrm{B}}\left(\mathbf{q}, l_{0}\right)=\mathbf{f}_{e}^{\mathrm{B}}\left(\mathbf{q}, l_{0}\right)+\mathbf{f}_{g}^{\mathrm{B}}\left(l_{0}\right)
$$

\subsection{Boundary conditions and solution}

Figure (5) shows a model of the catenary. The ANC beam element is used for the messenger wire (blue) and the contact wire (red). The droppers, the stitch wire and the registration arm are modeled as bar elements.

The following Dirichlet boundary conditions are imposed in the model: 

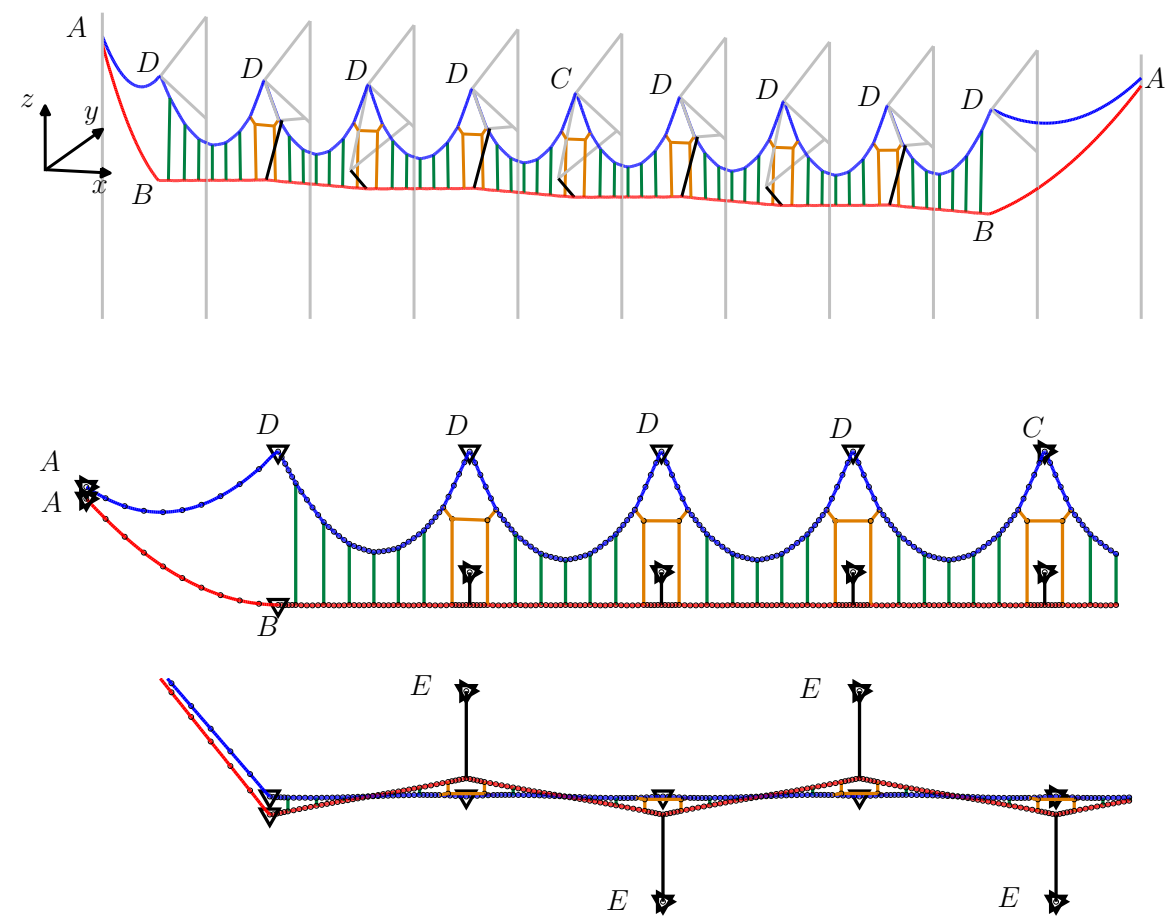

Figure 5: Boundary conditions in the finite element model.

- Label A in Figure (5). The initial and final nodes of the contact and messenger wires are restricted in all directions.

- Label B. The nodes of the contact wire located in the second and second to last masts are restricted in $y$ and $z$

- Label $\mathrm{C}$ is the midpoint anchor. The node of the messenger wire located in the central mast is constrained in all directions.

- Label D. The nodes of the messenger wire connected with the brackets are restricted in $y$ and $z$. The vertical constraint can be replaced by a spring element.

- Label E. The fixed nodes of the registration arm are constrained in all directions. 
The equilibrium configuration of the catenary is the solution of the nonlinear system obtained after the standard assembly of element force vectors

$$
\mathbf{f}\left(\mathbf{q}, \alpha, l_{0}\right)=\bigcup\left(\mathbf{f}^{\mathrm{C}}\left(\mathbf{q}, l_{0}\right)+\mathbf{f}^{\mathrm{ST}}\left(\mathbf{q}, \alpha, l_{0}\right)+\mathbf{f}^{\mathrm{B}}\left(\mathbf{q}, l_{0}\right)\right)=0
$$

The union symbol here is used to denote the standard finite element assembly process. Assuming that the undeformed length of each element is known, Equation (17) can be solved using for example the Newton-Raphson method.

The discontinuous traction element has an advantage for solving the nonlinear problem using the Newton-Raphson method. The new coordinate $\alpha$ of each element depends only on the geometry of the element and the assembly process results in an independent equation. This equation can therefore be eliminate without assembly and without increasing size of the global system. On the other hand, some new calculations are performed during iterations to update the value of $\alpha$ for each element.

Note that the equilibrium position (Equation (17)) depends on the undeformed length of the elements, which is not generally known in the case of a catenary system. In the following section we propose two methods of computing these lengths.

\section{Initial configuration problem}

As pointed out above, the finite element model of the catenary must reproduce the constraints introduced during the assembly of the system. We thus propose a method of finding the position of each node and the initial length of each element in the mesh that fulfills these constraints, besides the force equilibrium equations. First, a finite element mesh of the system is defined taking into account the desired number of elements in each wire. A tentative initial configuration of the catenary and the reference length of the elements are computed by the analytical catenary equation. To pose the problem, we need to define the constraints to be fulfilled and the corresponding set of element lengths to be computed. As the number of unknowns must be equal to the number of equations, rather than defining the initial length of each element as unknown, we define some groups of elements whose initial length is modified by a factor $\kappa_{l}$. The factor $\kappa_{l}$ for each group is the unknown of the problem. The following groups of elements are defined for each constraint: 


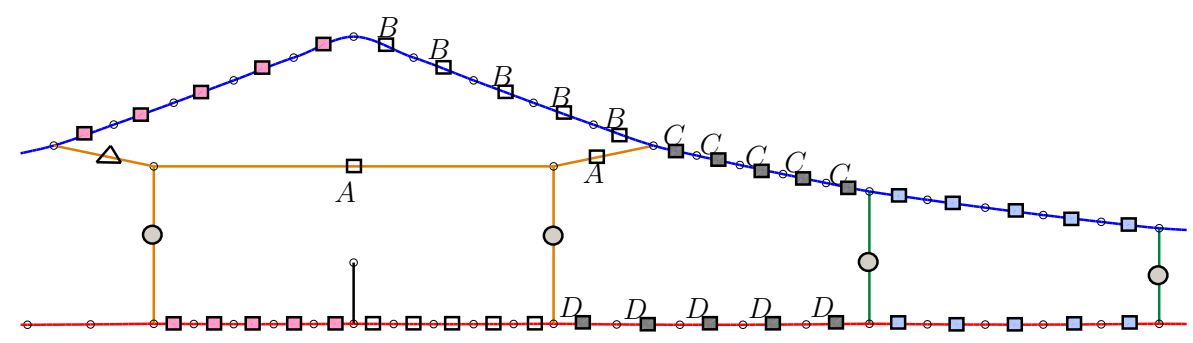

Figure 6: Groups of elements.

- Tension in the messenger wire: All the elements of the messenger wire in the first span form the group sharing the same length factor $\kappa_{l}$. This factor is used to impose the first constraint. If the catenary has a fixed point, the last span is also defined as a group with variable factor length on which the traction in the second part of the wire is imposed.

- Tension in the contact wire: All the elements in the first span of the contact wire belongs to a group.

- Tension in the stitch wire: Figure (6) shows a schematic diagram of the stitch wire. The element identified by a triangle is the group used to impose the traction in this wire.

- Position of the stitch wire: The longitudinal position of the connecting point of stitch wire and droppers can be imposed by defining the two groups of elements shown in Figure (6) by a square and label A.

- Position of the nodal mast: The group of elements between the bracket and the stitch wire connection is chosen to impose the longitudinal position of the messenger wire/bracket connection. This is shown in Figure (6) by a square and label B.

- Position of the droppers: The set of elements between two droppers in the messenger and contact wires forms the groups that impose the longitudinal position of the droppers (square symbol in Figure (6) and labels $\mathrm{C}$ and $\mathrm{D}$ ).

- Height of the contact wire. Each dropper is a group that controls the height of the contact wire (circle in Figure (6)). 


\subsection{Pointwise constraint method}

The first method of obtaining a static configuration of the catenary consist of solving a set of equations that include the equilibrium of internal and external forces (Equation (17)) and the following constraint equations:

- Traction in the messenger, contact and stitch wires. The internal force in the node in which the traction is imposed must be equal to the desired value $T$, so the equation can be written as:

$$
c_{\mathrm{I}}\left(\mathbf{q}, \kappa_{l}\right)=\mathrm{f}_{\mathrm{e}}^{\mathrm{ANC}}{ }_{x}^{2}+\mathrm{f}_{\mathrm{e}}^{\mathrm{ANC}}{ }_{y}^{2}+\mathrm{f}_{\mathrm{e}}^{\mathrm{ANC}}{ }_{z}^{2}-T^{2}=0
$$

where $\mathrm{f}_{\mathrm{e}}^{\mathrm{ANC}}{ }_{i}$ is the $i$ component of the internal elastic force at a given node.

- Global $x$ position of the connection points of droppers with messenger and contact wires, of brackets with the messenger wire and registration arm with contact wire. In this case the equation to be fulfilled is simply:

$$
c_{\mathrm{II}}(\mathbf{q})=\mathrm{q}_{x}-v_{x}=0
$$

where $v_{x}$ is the desired value of position.

- Height of the contact wire. The height of the contact wire $h_{c}$ is imposed at each contact wire/dropper connection. The equation can be written as:

$$
c_{\mathrm{III}}(\mathbf{q})=\mathrm{q}_{z}-h_{c}=0
$$

Equilibrium equations (17) together with the constraints (18), (19) and (20) forms a system of non-linear equations:

$$
\left.\begin{array}{r}
\mathbf{f}\left(\mathbf{q}, \kappa_{l} l_{0}\right)=0 \\
\mathbf{c}\left(\mathbf{q}, \kappa_{l} l_{0}\right)=0
\end{array}\right\}
$$

The system can be solved by the Newton-Raphson method to obtain the position of each node $\mathbf{q}$ and the factor $\kappa_{l}$ of each group. 


\subsection{Height optimization method}

The second method proposed for finding the initial configuration of the catenary is a minimization problem under constraints. The functional to be minimized is the quadratic difference between the height of the contact wire a the desired value $h_{c}$ along a given catenary path. The imposed constraints are the traction in messenger, contact and stitch wires $\mathbf{c}_{\mathrm{I}}$ (Equation (18)) and the position of connection points $\mathbf{c}_{\mathrm{II}}$ (Equation (19)) besides the equilibrium equations (Equation (17)). Formally, the problem can be written as:

$$
\begin{aligned}
& \min \frac{1}{2} \sum_{\forall p_{g}}\left(q_{z}\left(p_{g}\right)-h_{c}\right)^{2} \\
& \text { subject to } \\
& \quad \mathbf{f}\left(\mathbf{q}, \kappa_{l} l_{0}\right)=0 \\
& \quad \mathbf{c}_{\mathrm{I}}\left(\mathbf{q}, \kappa_{l} l_{0}\right)=0 \\
& \quad \mathbf{c}_{\mathrm{II}}(\mathbf{q})=0
\end{aligned}
$$

where $\mathrm{q}_{z}\left(p_{g}\right)$ is the global coordinate $z$ of the contact wire computed at a number of points $p_{g}$ along the path of the catenary. The problem of Equation (22) can be solved using the Lagrange multiplier method. Additional variables, the Lagrange multipliers $\lambda_{i}$, are introduced and the minimization problem can be written as the optimization of the following functional:

$$
\mathcal{L}\left(\mathbf{q}, \kappa_{l} l_{0}, \lambda\right)=\frac{1}{2}\left(\sum_{\forall p_{g}}\left(\mathrm{q}_{z}\left(p_{g}\right)-h_{c}\right)^{2}+\lambda_{\mathrm{III}} \mathbf{f}+\lambda_{\mathrm{I}} \mathbf{c}_{\mathrm{I}}+\lambda_{\mathrm{II}} \mathbf{c}_{\mathrm{II}}\right)
$$

Taking the variation of the functional of Equation (23) with respect to all variables, that is, the global coordinates $\mathbf{q}$, the factor of length $\kappa_{l}$ and the Lagrange multipliers $\lambda_{i}$, we obtain the following system of nonlinear equations

$$
\begin{aligned}
& \sum_{\forall p_{g}}\left(\mathrm{q}_{z}\left(p_{g}\right)-h_{c}\right) \frac{\partial \mathrm{q}_{z}\left(p_{g}\right)}{\partial \mathbf{q}}+\lambda_{\mathrm{III}} \frac{\partial \mathbf{f}}{\partial \mathbf{q}}+\lambda_{\mathrm{I}} \frac{\partial c_{\mathrm{I}}}{\partial \mathbf{q}}+\lambda_{\mathrm{II}} \frac{\partial c_{\mathrm{II}}}{\partial \mathbf{q}}=0 \\
& \lambda_{\mathrm{III}} \frac{\partial \mathbf{f}}{\partial l_{0}}+\lambda_{\mathrm{I}} \frac{\partial c_{\mathrm{I}}}{\partial l_{0}}+\lambda_{\mathrm{II}} \frac{\partial c_{\mathrm{II}}}{\partial l_{0}}=0 \\
& \mathbf{f}\left(\mathbf{q}, \kappa_{l} l_{0}\right)=0 \\
& \mathbf{c}_{\mathrm{I}}\left(\mathbf{q}, \kappa_{l} l_{0}\right)=0 \\
& \mathbf{c}_{\mathrm{II}}(\mathbf{q})=0
\end{aligned}
$$




\section{Results}

The methods described above were used to compute the equilibrium configuration and the length of the droppers for some reference problems. The results are compared with the length of droppers obtained from the literature for the same configurations. The method of pointwise constraint of Section (4.1) is used for the these examples. An example of a real catenary is also presented and the methods of Sections (4.1) and (4.2) are compared.

\subsection{Reference Catenary 1}

The first reference catenary consist of a single span with two droppers and no stitch wire. This catenary was proposed in [27] as a benchmark problem to test the dynamic response of the pantograph-catenary interaction. The data used in this problem are presented in Table (1) and the wire element data used in the proposed method are shown in Table (2). The same geometry was analyzed in [28], in which a catenary element was used. One of the differences between the $\mathrm{ANCF}$ element and the catenary element is in the computation of the internal force. In the first, case axial and bending deformations are included, whilst the catenary element assumes rigid behavior in axial direction and neglects the strain due to bending.

\begin{tabular}{|l|l|}
\hline Span length $L_{s}$ & $20 \mathrm{~m}$ \\
\hline Encumbrance $H_{s}$ & $1.0 \mathrm{~m}$ \\
\hline Number of droppers & 2 \\
\hline Longitudinal position & $1: \quad x=5.5$ \\
of droppers $(\mathrm{m})$ & $2: \quad x=14.5$ \\
\hline
\end{tabular}

Table 1: Geometrical data for the reference catenary 1

\begin{tabular}{|l|l|l|l|}
\hline & Contact & Messenger & Droppers \\
\hline Axial stiffness $E A(\mathrm{MN})$ & 13.053 & 6.667 & 1.711 \\
\hline Bending stiffness $E J\left(\mathrm{Nm}^{2}\right)$ & 150.0 & 0.0 & - \\
\hline Mass/unit length $(\mathrm{kg} / \mathrm{m})$ & 1.068 & 0.60 & 0.14 \\
\hline Tension $(\mathrm{kN})$ & 15 & 15 & - \\
\hline Clamp mass $(\mathrm{kg})$ & 0.25 & 0.25 & - \\
\hline
\end{tabular}

Table 2: Material data for Reference Catenary 1 
The reference configuration obtained for this catenary is shown in Figure (7). The undeformed length of the droppers is found to be $L_{d}=0.9540$ $\mathrm{m}$. This value is in agreement with the value obtained in reference [27] $L_{d}=0.95 \mathrm{~m}$ or reference [28] $L_{d}=0.9579 \mathrm{~m}$. Despite the differences in the formulations the results are very similar. For this catenary the influence of axial and bending deformations can therefore be said to be negligible.

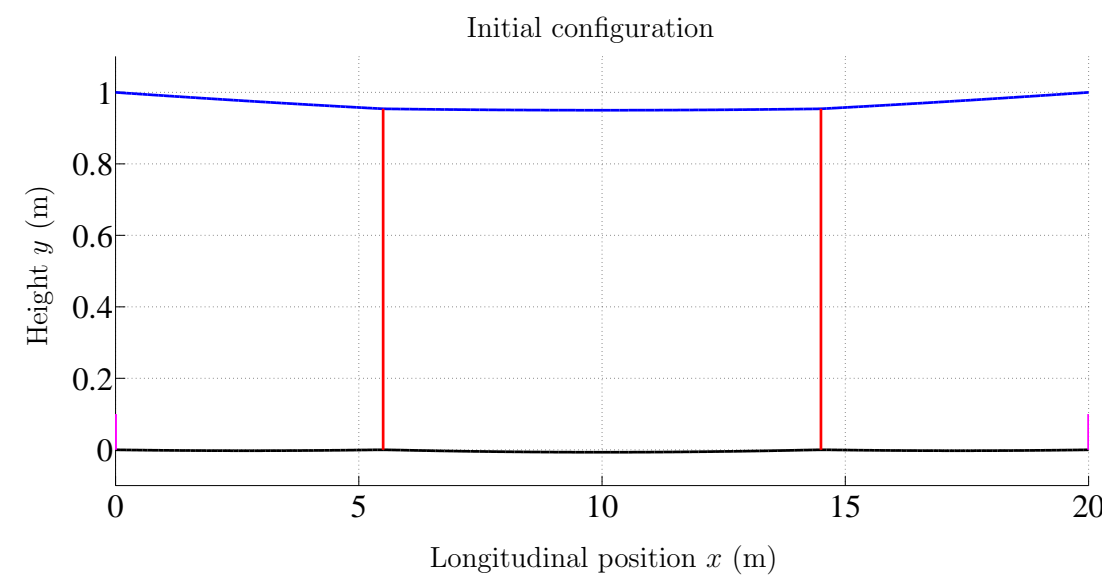

Figure 7: Equilibrium initial configuration of Reference Catenary 1.

The influence of the finite element discretization error was analyzed. The length of droppers was computed with different meshes. The number of elements of the contact and messenger wires range from 6 to 12. The results are shown in Table (3) for different combinations of elements in the contact wire and messenger wires. As can be observed, the influence of the number of elements is at most a few millimeters.

Messenger wire

\begin{tabular}{|c|c|c|c|c|c|c|}
\hline & $\begin{array}{c}\text { Number of } \\
\text { elements }\end{array}$ & 6 & 12 & 24 & 48 & 96 \\
\hline \multirow{5}{*}{ 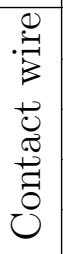 } & 6 & 0.9543 & 0.9537 & 0.9534 & 0.9533 & 0.9532 \\
\hline & 12 & 0.9561 & 0.9548 & 0.9542 & 0.9539 & 0.9537 \\
\hline & 24 & 0.9550 & 0.9544 & 0.9542 & 0.9540 & 0.9540 \\
\hline & 48 & 0.9551 & 0.9545 & 0.9542 & 0.9541 & 0.9540 \\
\hline & 96 & 0.9551 & 0.9545 & 0.9543 & 0.9541 & 0.9540 \\
\hline
\end{tabular}

Table 3: Undeformed length of the droppers $(\mathrm{m})$ for different finite element meshes. 


\subsection{Reference Catenary 2}

The second example was proposed in [30]. This problem has two configurations: with and without presag. The authors proposed a method of computing the initial configuration of the catenary and provide the lengths of the droppers. They used a finite element model with the Euler-Bernouilli beam element. The data pertaining to the simulation of this problem are presented in Tables (4) and (5). Note that we have introduced the axial stiffness to allow for axial deformation of wires, as data was lacking in reference [30]. The droppers are assumed to have a constant stiffness. Dropper mass is concentrated in the clamps.

\begin{tabular}{|l|l|l|l|}
\hline Span length $L_{s}$ & $50 \mathrm{~m}$ & Bracket stiffness & $2 \cdot 10^{6} \mathrm{~N} / \mathrm{m}$ \\
\hline Encumbrance $H_{s}$ & $0.96 \mathrm{~m}$ & Registration arm stiffness & $213 \mathrm{~N} / \mathrm{m}$ \\
\hline Dropper stiffness & $10^{5} \mathrm{~N} / \mathrm{m}$ & Registration arm mass & $2.6 \mathrm{~kg}$ \\
\hline Longitudinal position & $1: \quad x=2.5$ & $6: \quad x=27.5$ & \\
of droppers $(\mathrm{m})$ & $2: \quad x=7.5$ & $7: \quad x=32.5$ & \\
& $3: \quad x=12.5$ & $8: \quad x=37.5$ & \\
& $4: \quad x=17.5$ & $9: \quad x=42.5$ & \\
& $5: \quad x=22.5$ & $10: \quad x=47.5$ & \\
\hline
\end{tabular}

Table 4: General data for Reference Catenary 2

\begin{tabular}{|l|l|l|}
\hline & Contact & Messenger \\
\hline Axial stiffness $E A(\mathrm{MN})$ & 12.063 & 6.722 \\
\hline Bending stiffness $E J\left(\mathrm{Nm}^{2}\right)$ & 130.0 & 29.0 \\
\hline Mass/unit length $(\mathrm{kg} / \mathrm{m})$ & 0.987 & 0.605 \\
\hline Tension $(\mathrm{kN})$ & 12 & 12 \\
\hline Clamp mass $(\mathrm{kg})$ & 0.2 & 0.2 \\
\hline
\end{tabular}

Table 5: Material data for Reference Catenary 2

Figures (8) and (9) show the initial configuration of the catenary with presag. The length of the droppers is compared in Table (6) for the catenaries with and without presag. The results show a good agreement in all computed values, even though the type of element and axial behavior of the wires are different and a different method is used to compute the equilibrium position. 


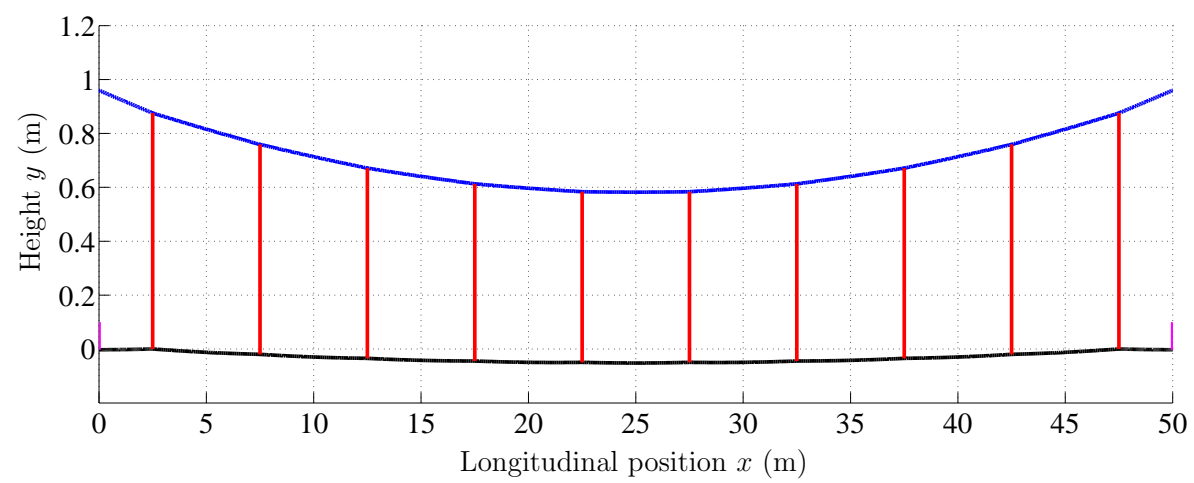

Figure 8: Initial equilibrium configuration of Reference Catenary 2.

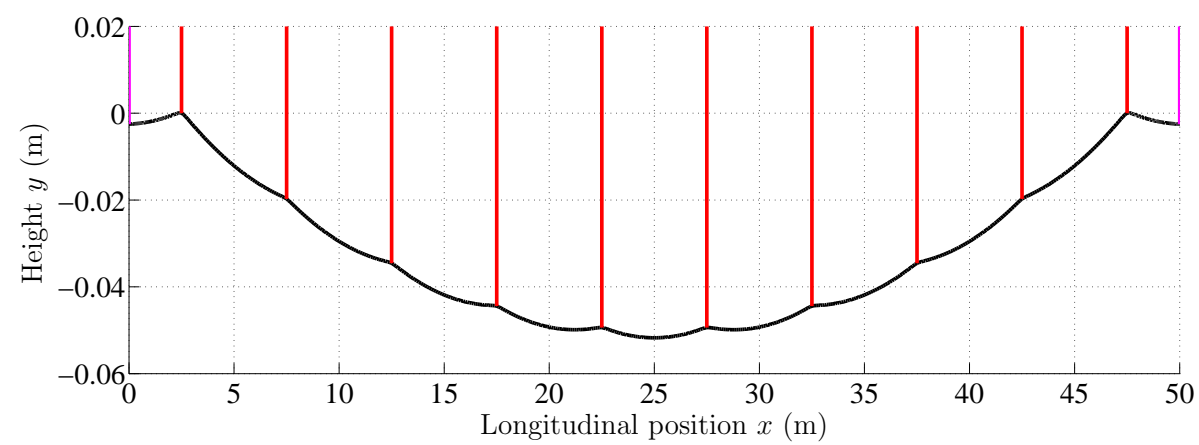

Figure 9: Initial equilibrium configuration of the contact wire in Reference Catenary 2.

Without presag

\begin{tabular}{|l|c|c|c|c|c|}
\hline Dropper & 1 & 2 & 3 & 4 & 5 \\
\hline Reference [30] & 0.875 & 0.738 & 0.636 & 0.567 & 0.533 \\
\hline Present paper & 0.8751 & 0.7386 & 0.6361 & 0.5678 & 0.5336 \\
\hline \multicolumn{6}{|c|}{ With presag } \\
\hline Dropper & 1 & 2 & 3 & 4 & 5 \\
\hline Reference [30] & 0.875 & 0.778 & 0.705 & 0.657 & 0.633 \\
\hline Present paper & 0.8747 & 0.7784 & 0.7056 & 0.6571 & 0.6328 \\
\hline
\end{tabular}

Table 6: Length of droppers (m) for Reference Catenary 2

\subsection{High speed train catenary}

The last example is a real catenary with 10 central spans in addition to four spans for the transitions. The properties of the catenary are detailed in 
Tables (7) and (8). In this case we compare the two methods proposed to solve the initial configuration problem (Sections (4.1) and (4.2)). A curved track path with radius $R=20000 \mathrm{~m}$ is also generated and the lengths of droppers are compared with those obtained from a straight path. To solve problems with curved paths, the element formulation is modified with the appropriate coordinate transforms to impose the equilibrium of equations as well as the other constraints in a local coordinate system. A schematic diagram of the two tracks is shown in Figure (10).

\begin{tabular}{|l|l|l|l|}
\hline Span length $L_{s}$ & $65 \mathrm{~m}$ & Bracket stiffness & $2 \cdot 10^{6} \mathrm{~N} / \mathrm{m}$ \\
\hline Encumbrance $H_{s}$ & $1.3 \mathrm{~m}$ & Stitch wire length & $18 \mathrm{~m}$ \\
\hline Stagger messenger & $0 \mathrm{~m}$ & Stagger contact & $\pm 0.2 \mathrm{~m}$ \\
\hline Clamp messenger & $\pm 0.21 \mathrm{~kg}$ & Clamp contact & $\pm 0.21 \mathrm{~kg}$ \\
\hline Longitudinal position & $1: \quad x=6$ & Registration & \\
of droppers (m) & $2: \quad x=15.48$ & arm length & $1.15 \mathrm{~m}$ \\
& $3: \quad x=24.18$ & & \\
& $4: \quad x=32.5$ & & \\
& $5: \quad x=40.82$ & & \\
& $6: \quad x=49.52$ & & \\
& $7: \quad x=59$ & & \\
\hline
\end{tabular}

Table 7: General data of the real catenary problem

\begin{tabular}{|l|c|c|c|c|}
\hline & $\begin{array}{c}\text { Mass } / \text { unit } \\
\text { length }(\mathrm{kg} / \mathrm{m})\end{array}$ & $\begin{array}{c}\text { Axial stiffness } \\
E A(\mathrm{MN})\end{array}$ & $\begin{array}{c}\text { Bending stiffness } \\
E J\left(\mathrm{Nm}^{2}\right)\end{array}$ & $\begin{array}{c}\text { Tension } \\
(\mathrm{kN})\end{array}$ \\
\hline Messenger wire & 0.864 & 10.42 & 103.7 .8 & 15.75 \\
\hline Contact wire & 1.374 & 18 & 198.4 & 31.5 \\
\hline Stitch wire & 0.315 & 3.605 & - & 3.5 \\
\hline Droppers & 0.315 & 3.605 & - & - \\
\hline Registration arm & 0.773 & $72.2 \cdot 10^{-3}$ & - & - \\
\hline
\end{tabular}

Table 8: Material properties for the real catenary problem

The length of the droppers in three cases are shown in Table (9). The first case involves a straight catenary in which the height of the contact wire is imposed pointwise in the position at which the wire is connected to droppers and registration arms. The second case is a catenary with a curved track and pointwise constraints. In the last case the optimization method of Section 

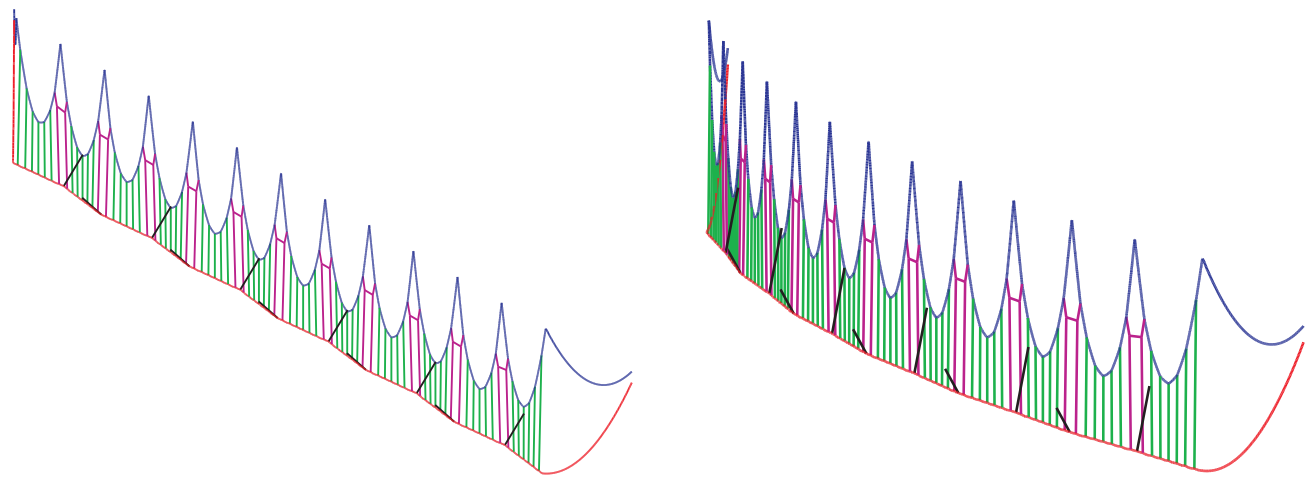

Figure 10: Initial configuration of the real catenary for straight and curved track paths.

\begin{tabular}{|l|c|c|c|c|c|c|c|}
\hline Dropper & 1 & 2 & 3 & 4 & 5 & 6 & 7 \\
\hline Straigth track & 0.7036 & 0.6130 & 0.4511 & 0.4001 & 0.4511 & 0.6130 & 0.7036 \\
\hline Curved track 1 & 0.7025 & 0.6131 & 0.4511 & 0.4000 & 0.4510 & 0.6129 & 0.7025 \\
\hline Optimization & 0.6904 & 0.6028 & 0.4432 & 0.3922 & 0.4432 & 0.6028 & 0.6904 \\
\hline
\end{tabular}

Table 9: Length of droppers $(\mathrm{m})$ for high speed train catenary

(4.2) is used for a straight catenary. Practically the same results are obtained for both straight and curved tracks, due to the large radius of the curve.

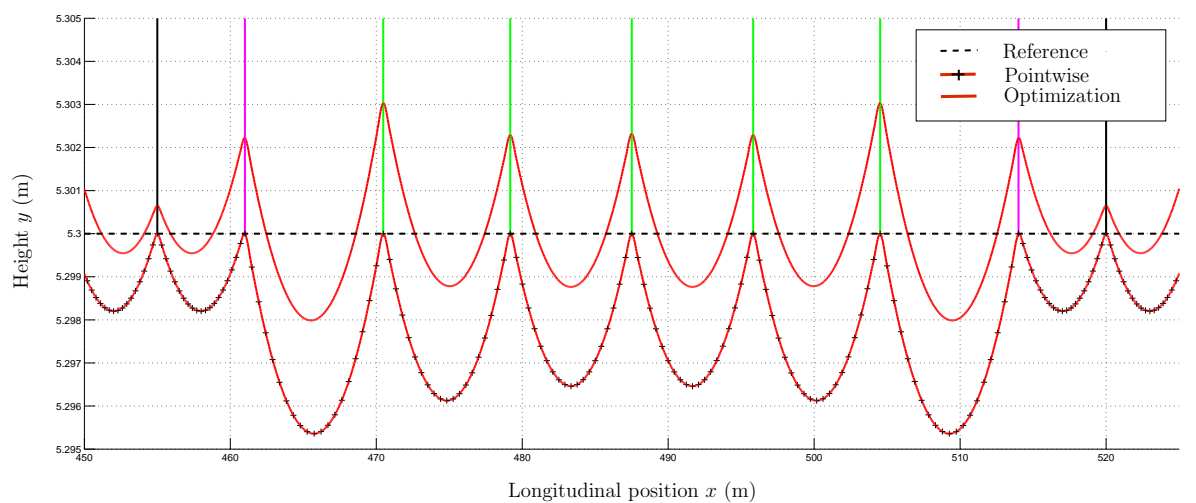

Figure 11: Position of the contact wire.

As can be observed, the length of the droppers using the optimization method is slightly less than that obtained with the pointwise constraint method. This can be explained by looking at Figure (11), where the po- 
sition of the contact wire is plotted for a single span in both cases. The reference height is $5.3 \mathrm{~m}$, which is attained exactly at droppers/contact wire connection point using the pointwise constraint method. A higher position is obtained for the optimization method since the reference height is imposed over the whole span.

\section{Conclusions}

This paper presents two methods of shape-finding applied to the finite element model of a catenary system, which take into account for the constraints imposed during the stringing of the catenary In the first method the height constraint in the contact wire is imposed at the points at which the wire is connected to the droppers. In the second method an optimization problem is solved. The objective function to be minimized is the difference in the height of the contact wire with respect to a reference value. Both methods were applied to find the initial configuration of some reference problems in the bibliography and a good agreement was obtained.

\section{Acknowledgements}

The authors wish to thank Generatitat Valenciana for the financial support received in the framework of the Programme PROMETEO 2012/023.

\section{References}

[1] G. Poetsch., J. Evans, R. Meisinger, W. Kortam, W. Baldauf, A. Veitl, J. Wallaschek, Pantograph / catenary dynamics and control, Vehicle System Dynamics 28 (2-3) (1997) 159-195. doi:10.1080/00423119708969353.

[2] J. Massat, Modélisation du comportement dynamique du couple pantographe-caténaire, Ph.D. thesis, Laboratoire de Tribologie et Dynamique des Systèmes, Ecole Centrale de Lyon (2007).

[3] J. Massat, J. Laine, A. Bobillot, Pantograph-catenary dynamic simulation, in: Proceedings of the IAVSD Conference, 2004.

[4] J. Massat, A. Bobillot, J. Laine, Robust methods for detecting defects in overhead contact line based on simulation results, in: .-. June (Ed.), III ECCOMAS Conference, Lisbon (Portugal), 2006. 
[5] J. Ambrosio, J. Pombo, F. Rauter, M. Pereira, Multiple pantograph interaction with catenaries in high-speed trains, in: M. 18-22 (Ed.), Proceedings of the 8th World Congress on Railway Research, Seoul (Korea), 2008.

[6] F. G. Rauter, J. Pombo, J. Ambrósio, J. Chalansonnet, A. Bobillot, M. S. Pereira, Contact model for the Pantograph-Catenary interaction, Journal of System Design and Dynamics 1 (2007) 447-457. doi:10.1299/jsdd.1.447.

[7] J. Pombo, J. Ambrósio, Influence of pantograph suspension characteristics on the contact quality with the catenary for high speed trains, Computers \& Structures 110-111 (2012) 32-42. doi:10.1016/j.compstruc.2012.06.005.

[8] A. Collina, S. Bruni, Numerical simulation of pantograph-overhead equipment interaction, Vehicle System Dynamics 38 (4) (2002) 261-291.

[9] A. Collina, A. L. C. M. Carnevale, Effect of collector deformable modes in pantograph-catenary dynamic interaction, Proceedings of the Institution of Mechanical Engineers, Part F: Journal of Rail and Rapid Transit 223 (1) (2009) 1-14.

[10] J. Pombo, J. Ambrosio, M. Pereira, F. Rauter, A. Collina, A. Facchinetti, Influence of the aerodynamic forces on the pantographcatenary system for high-speed trains, Vehicle System Dynamics 47 (11) (2009) 1327-1347.

[11] C. Vera, B. Suarez, J. Paulin, P. Rodrguez, Simulation model for the study of overhead rail current collector systems dynamics focused on the design of a new conductor rail, Vehicle System Dynamics 44 (8) (2006) 595-614.

[12] Y. Cho, Numerical simulation of the dynamic responses of railway overhead contact lines to a moving pantograph considering a nonlinear dropper, Journal of Sound and Vibration 315 (2008) 433-454.

[13] J. Benet, F. Cuartero, T. Rojo, A tool to calculate mechanical forces on railway catenary, in: Seventh International Conference on Computer in Railways, COMPRAIL-2000, Bolonia (Italy), 2000, pp. 437-451. 
[14] A. Alberto, J. Benet, E. Arias, D. Cebrian, T. Rojo, F. Cuartero, A high performance tool for the simulation of the dynamic pantographcatenary interaction, Mathematics and Computers in Simulation 79 (3) (2008) 652 - 667. doi:10.1016/j.matcom.2008.04.016.

[15] J. Seo, A. S. Kim, I. Jung, T. Park, J. Mok, Y. Kim, J. Chai, Dynamic analysis of a pantograph-catenary system using absolute nodal coordinates, Vehicle System Dynamics 44 (8) (2006) 615-630.

[16] J. Gerstmayr, A. Shabana, Analysis of thin beams and cables using the absolute nodal co-ordinate formulation, Nonlinear Dynamics 45 (1-2) (2006) 109-130. doi:10.1007/s11071-006-1856-1.

[17] A. Shabana, Computer implementation of the absolute nodal coordinate formulation for flexible multibody dynamics, Nonlinear Dynamics 16 (1998) 293-306.

[18] M. Berzeri, A. Shabana, Development of simple models for the elastic forces in the absolute nodal co ordinate formulation, Journal of Sound and Vibration 235 (4) (2000) 539-565.

[19] F. Rauter, J. Pombo, J. Ambrosio, M. Pereira, Multibody modeling of pantographs for pantograph-catenary interaction, in: P. Eberhard (Ed.), IUTAM Symposium on Multiscale Problems in Multibody System Contacts, Vol. 1 of IUTAM Bookseries, Springer Netherlands, 2007, pp. 205-226. doi:10.1007/978-1-4020-5981-0_20.

[20] J. Ambrosio, F. Rauter, J. Pombo, M. Pereira, A flexible multibody pantograph model for the analysis of the catenarypantograph contact, in: K. Arczewski, W. Blajer, J. Fraczek, M. Wojtyra (Eds.), Multibody Dynamics, Vol. 23 of Computational Methods in Applied Sciences, Springer Netherlands, 2011, pp. 1-27. doi:10.1007/978-90-481-9971-6_1.

[21] G. Tibert, Numerical analyses of cable roof structures, Ph.D. thesis, Department of Structural Engineering, Royal Institute of Technology Stockholm (1999).

[22] B. B. J. H. Argyris, T. Angelopoulos, A general method for the shape finding of lightweight tension structures, Computer Methods in Applied Mechanics and Engineering 3 (1974) 135-149. 
[23] M. Barnes, Form-finding and analysis of prestressed nets and membranes, Computers \& Structures 30 (3) (1988) 685-695.

[24] H. Scheck, The force density method for the form-finding and computation of general networks, Computer Methods in Applied Mechanics and Engineering 3 (1974) 115-134.

[25] P. P. R.M.O. Pauletti, The natural force density method for the shape finding of taut structures, Computer Methods in Applied Mechanics and Engineering 197 (2008) 4419-4428.

[26] B. Descamps, R. F. Coelho, L. Ney, P. Bouillard, Multicriteria optimization of lightweight bridge structures with a constrained force density method, Computers \& Structures 89 (2011) 277-284.

[27] M. Arnold, B. Simeon, Pantograph and catenary dynamics: A benchmark problem and its numerical solution, Applied Numerical Mathematics 34 (4) (2000) 345-362.

[28] O. López-García, A. Carnicero, V. Torres, Computation of the initial equilibrium of railway overheads based on the catenary equation, Engineering Computations 28 (2006) 1387-1394.

[29] G. Teichelmann, M. Schaub, B. Simeon, Modelling and simulation of railway cable systems, Journal of Applied Mathematics and Mechanics (ZAMM) 85 (12) (2005) 864877.

[30] Y. Cho, K. Lee, Y. Park, B. Kang, K. Kim, Influence of contact wire pre-sag on the dynamics of pantographrailway catenary, International Journal of Mechanical Sciences 52 (2010) 1470-1490.

[31] M. Yang, Z. Chen, X. Hua, A new two-node catenary cable element for the geometrically non-linear analysis of cable-supported structures, Journal of Mechanical Engineering Science 224 (6) (2010) 1173-1183.

[32] H. Jayaraman, W. Knudson, A curved element for the analysis of cable structures, Computers \& Structures 14 (3) (1981) 325-333.

[33] R. Karoumi, Some modeling aspects in the nonlinear finite element analysis of cable supported bridges, Computers \& Structures 71 (4) (1999) 397-412. 
[34] A. Andreu, L. Gil, P. Roca, A new deformable catenary element for the analysis of cable net structures, Computers \& Structures 84 (29) (2006) 1882-1890.

[35] J. Gerstmayr, H. Irschik, On the correct representation of bending and axial deformation in the absolute nodal coordinate formulation with an elastic line approach, Journal of Sound and Vibration 318 (2008) 461487.

[36] J.-H. Lee, T.-W. Park, Development of a three-dimensional catenary model using cable elements based on absolute nodal coordinate formulation, Journal of Mechanical Science and Technology 26 (12) (2012) 3933-3941. doi:10.1007/s12206-012-0892-7.

URL http://dx.doi.org/10.1007/s12206-012-0892-7

[37] W. Ren, M. Huang, W. Hu, A parabolic cable element for static analysis of cable structures, Engineering Computations 25 (4) (2008) 366-384.

[38] I. Romero, A comparison of finite elements nonlinear beams: the absolute nodal coordinate and geometrically exact formulations, Multibody System Dynamics 20 (2008) 51-68. 